\title{
Differences in the dense gas between type 1 and type 2 Seyferts ${ }^{\star}$
}

\author{
S.J. Curran \\ Onsala Space Observatory, Chalmers University of Technology, S-439 92 Onsala, Sweden \\ e-mail: sjc@oso.chalmers.se
}

Received December 1, 1999; accepted February 28, 2000

\begin{abstract}
We have used observations of the $\mathrm{CO} J=1 \rightarrow$ 0 transition in a sample of 20 Seyfert galaxies in order to test the result of Heckman et al. (1989), who find that type 2 Seyferts have a higher molecular gas content than type 1 Seyferts. From our observational results alone, we possibly find that $L_{\mathrm{CO}} / L_{\mathrm{FIR}}(\mathrm{Sy} 2) \approx L_{\mathrm{CO}} / L_{\mathrm{FIR}}(\mathrm{Sy} 1)$ for $L_{\mathrm{FIR}} \sim 10^{11} L_{\odot}$ Seyferts, but $L_{\mathrm{CO}} / L_{\mathrm{FIR}}(\mathrm{Sy} 2) \approx$ $3 L_{\mathrm{CO}} / L_{\mathrm{FIR}}(\mathrm{Sy} 1)$, when we use all of our sample $\left(L_{\mathrm{FIR}} \sim\right.$ $\left.10^{10}-10^{11} L_{\odot}\right)$. The results suggest that star-burst activity is most dominant in the $\left(L_{\mathrm{FIR}} \sim 10^{10} L_{\odot}\right)$ type 2 Seyferts, and the relative contribution of this activity to the FIR flux (compared to the AGN) may decrease with increasing $L_{\mathrm{FIR}}$.

Also, in order to test whether the Seyfert classes differ in their molecular gas distributions, we have used a simple model to estimate the inclination of the sub-kpc scale molecular ring, and, upon comparison with the observed spectra, conclude that the ring is generally aligned with the galactic disc, with the largest deviations from this being exhibited by the type 1 Seyferts. With regard to the nucleus, for most of the sample, the pc-scale obscuring torus appears to be approximately (within $\approx 30^{\circ}$ ) aligned with the molecular ring for both Seyfert classes.
\end{abstract}

Key words: galaxies: Seyfert — galaxies: abundances galaxies: structure - galaxies: evolution

\section{Introduction}

Seyfert galaxies are divided into two main classes according to their optical properties. The main difference being that type 1 Seyferts (Sy1s) exhibit a broad line region, in addition to the narrow line region, which is evident in both the classes. According to the currently favoured scenario,

Send offprint requests to: S. Curran

* Based on results collected at the European Southern Observatory, La Silla, Chile and Onsala Space Observatory, Sweden. the Seyfert nucleus is surrounded by a dusty torus shaped region and the observed properties are determined solely by the orientation of the torus relative to the observers line of sight to the nucleus (Antonucci 1993). In type 1 nuclei, the axis of the torus is close to the line of sight and one observes a naked AGN directly with its associated broad line region in full view. In type 2 nuclei, the orientation of the dusty torus is such that it shields the nucleus from view and only the more extended narrow line clouds are observed. It is not clear what difference this would make to the observed molecular properties of the galaxies; for the Sy2 galaxy NGC 4258 the CO is widely distributed and appears to have no connection to the nuclear activity. Contrary to this, Heckman et al. (1989) have found that in Sy2s, the observed ratio of the CO luminosity, $L_{\mathrm{CO}}$, as a fraction of the blue luminosity, is a factor of two greater than for Sy1s. An enhanced CO/blue luminosity ratio in Sy2s is also observed by Sahai et al. (1991). Since most of the blue continuum is generally believed to emanate from the accretion disc (e.g. Peterson 1997), which is obscured in Sy2s, these studies have possibly failed to take into account the fact that, on average, the Sy2s selected on this basis will tend to be more luminous than the Sy1s. In this paper we test their results by comparing the $\mathrm{CO}$ luminosities with respect to the far infrared (FIR) luminosities, as the latter quantity is expected to be a more isotropic parameter than the blue luminosity.

Another possible distinction between the two main Seyfert classes is the fact that, as well as the difference in orientation of the dusty obscuring torus, there may exist differences on how the molecular gas is distributed. While McLeod \& Rieke (1995); Maiolino \& Rieke (1995) find that the galactic disc is aligned with the $\gtrsim 100 \mathrm{pc}-$ scale molecular ring $^{1}$ (Sy2 nuclei tend to lie in edge-on

\footnotetext{
${ }^{1}$ Suspected to exist in all Seyfert galaxies e.g. (Myers \& Scoville 1987; Plante et al. 1991; Bergman et al. 1992; Irwin \& Sofue 1992; Kohno et al. 1996; Tacconi et al. 1996; Curran et al. 1998; Tacconi et al. 1999a). In fact such molecular rings may in all galaxies (Sofue 1991), including our own (Güsten 1989).
} 
galaxies and Sy1s in face-on systems), they find that Sy1 nuclei may be partially obscured by the ring, which could be misaligned with the pc-scale obscuring torus. However, Wilson \& Tsvetanov (1994); Capetti et al. (1996) find that the axis of the ionisation cone ${ }^{2}$, coincident with the axis of the obscuring torus which collimates the ionised outflow (Antonucci \& Miller 1985; Wilson et al. 1988; Tadhunter \& Tsvetanov 1989; Wilson \& Tsvetanov 1994; Baker \& Scoville 1998), also tends to be aligned with the host galaxy disc axis, thus placing the disc coplanar with the torus.

In this paper we use the observational results of the Northern and Southern Seyfert survey of Curran et al. (2000) in order to test the two main points outlined above, namely:

1. Statistical studies of the difference in dense gas abundances between type 1 and type 2 Seyferts.

2. Differences in the aspect of the gas distribution between type 1 and type 2 Seyferts.

\section{Statistical studies of the difference in dense gas abundances between type 1 and type 2 Seyferts}

In Table 1 we show the results of Curran et al. (2000). The source list is based upon the detections of Heckman et al. (1989) as when planning the survey it was our original intention to test the previous results. In the same way as Heckman et al. $(1989)^{3}$, the Seyferts are segregated into two main classes using the classification scheme of Maiolino \& Rieke (1995) (and Meurs \& Wilson 1984; Edelson 1987; Osterbrock \& Shaw 1988); where types $1,1.2$ and 1.5 constitute type 1 Seyferts (hereafter Sy1s) and types 1.8, 1.9 and 2 constitute type 2 Seyferts (hereafter Sy2s).

When we plot the CO luminosity against that of the FIR, Fig. 1, we see that apart from an anomalous point at $\approx 12, \approx 3.5$, the two linear fits would be close to parallel, although the intercept would be $\approx 0.4$ higher for the Sy2s making $\frac{L_{\mathrm{CO}}}{L_{\mathrm{FIR}}}(\mathrm{Sy} 2) \approx 3 \frac{L_{\mathrm{CO}}}{L_{\mathrm{FIR}}}(\mathrm{Sy} 1)^{4}$. Taking the average value of $L_{\mathrm{CO}} / L_{\mathrm{FIR}}$ for both the main classes, however, we find

$\frac{L_{\mathrm{CO}}}{L_{\mathrm{FIR}}}(\mathrm{Sy} 2) \approx \frac{L_{\mathrm{CO}}}{L_{\mathrm{FIR}}}(\mathrm{Sy} 1)$

$\approx 10^{-8} \mathrm{~K} \mathrm{~km} \mathrm{~s}^{-1} \mathrm{kpc}^{2} L_{\odot}^{-1}$. The anomalous point in Fig. 1 is in fact due to Mrk $273^{5}$ which has been noted

\footnotetext{
${ }^{2}$ Dust and ionised gas, which have accreted onto the AGN, being driven back outward in the direction of the jets by radiation pressure.

${ }^{3}$ Except in the case of NGC 5033 which they class as a Sy1, cf. Sy 1.9 (NASA/IPAC Extragalactic Database, Maiolino et al. 1997).

${ }^{4}$ For the Sy1s Fig. 1 gives $L_{\mathrm{CO}}=10^{-5} L_{\mathrm{FIR}}{ }^{0.75}$, and forcing a gradient of 1 gives $L_{\mathrm{CO}}=10^{-8} L_{\mathrm{FIR}} \mathrm{K} \mathrm{km} \mathrm{s}^{-1} \mathrm{kpc}^{2} L_{\odot}^{-1}$.

5 The one ultra-luminous infrared galaxy (ULIRG) of the Sy2 sample.
}

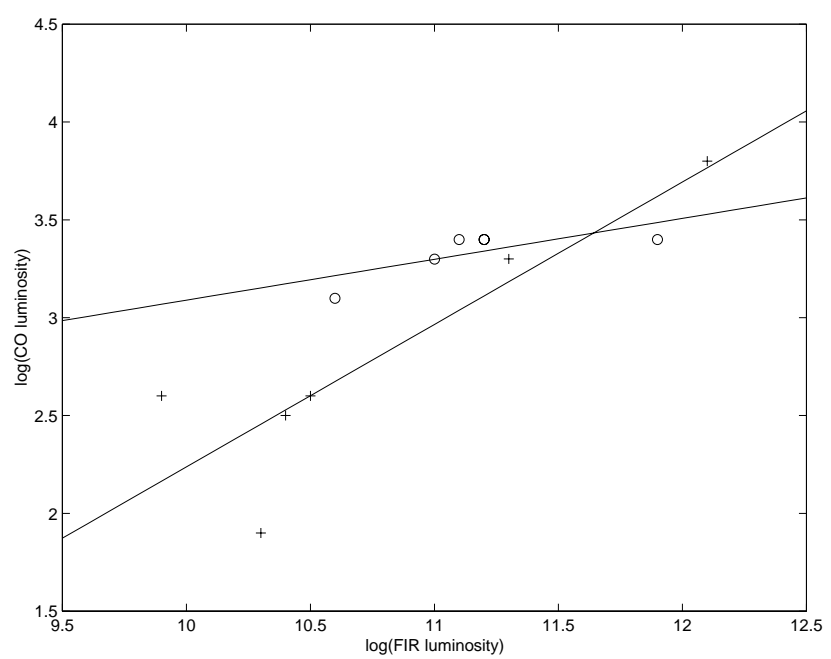

Fig. 1. $\log L_{\mathrm{CO}}\left[\mathrm{K} \mathrm{km} \mathrm{s}^{-1} \mathrm{kpc}^{2}\right]$ versus $\log L_{\mathrm{FIR}}\left[L_{\odot}\right]$ for the observed Sy1s (crosses) and Sy2s (circles) with recessional velocities of $v \gtrsim 4000 \mathrm{~km} \mathrm{~s}^{-1}$ : This velocity corresponds to a HPBW of $\approx 12 \mathrm{kpc}$ (at SEST, $\approx 10 \mathrm{kpc}$ at Onsala (OSO); assuming $\left.H_{0}=75 \mathrm{~km} \mathrm{~s}^{-1} \mathrm{Mpc}^{-1}\right)$, beyond which there is expected to exist little molecular gas (Maiolino et al. 1997), making us confident that we have sampled all of the $\mathrm{CO}$ in the faster receding sources

to have an unusually low $L_{\mathrm{CO}} / L_{\mathrm{HCN}}$ ratio (Curran et al. 2000).

Taking the average $L_{\mathrm{CO}} / L_{\mathrm{FIR}}$ ratios for all of the sample (i.e. including the global values), we find that

$\frac{L_{\mathrm{CO}}}{L_{\mathrm{FIR}}}(\mathrm{Sy} 2) \approx 3 \frac{L_{\mathrm{CO}}}{L_{\mathrm{FIR}}}(\mathrm{Sy} 1)$

$\approx 410^{-8} \mathrm{~K} \mathrm{~km} \mathrm{~s}^{-1} \mathrm{kpc}^{2} L_{\odot}^{-1}$. These results suggest two distinct possibilities:

1. Only use of the sources with $v \gtrsim 4000 \mathrm{~km} \mathrm{~s}^{-1}$ is valid, i.e. Eq. (1) holds. This agrees with the results of Maiolino et al. (1997), who compare the CO with blue luminosities and HI mass;

2. Use of all of the sources is valid, i.e. Eq. (2) holds. This gives a similar result to Heckman et al. (1989), who also use HI mass to obtain the same result as they get from the blue luminosities.

Since our results may be consistent with those of both Heckman et al. (1989) and Maiolino et al. (1997), where CO and blue luminosities are compared, they may suggest that the blue continuum is more isotropic than previously thought, i.e. arising from an additional source to the AGN (e.g. Fernandes \& Terlevich 1995).

Our findings could be compounded by the fact that (perhaps due to a selection effect), on average, $L_{\mathrm{FIR}}\left(v \gtrsim 4000 \mathrm{~km} \mathrm{~s}^{-1}\right.$ Seyferts $) \approx 15 L_{\mathrm{FIR}}(v \lesssim$ $4000 \mathrm{~km} \mathrm{~s}^{-1}$ Seyferts). From our sample we find that, on average, $L_{\mathrm{FIR}}(\mathrm{Sy} 2) \approx L_{\mathrm{FIR}}(\mathrm{Sy} 1)\left(\approx 210^{11} L_{\odot}\right)$ and so it appears as though the results are bias free, although the average FIR luminosity values are dominated by the ULIRGs Mrk231, Mrk 273 and Arp220 
Table 1. The observed luminosities of the sample. Sy refers to the main Seyfert type, $L_{\mathrm{CO}} 1 \rightarrow 0$ and $L_{\mathrm{HCN}} 1 \rightarrow 0$ refer to the luminosity over the HPBW for each respective transition, with $1 \sigma$ errors and upper limits $\left[\times 10^{3} \mathrm{~K} \mathrm{~km} \mathrm{~s}^{-1} \mathrm{kpc}^{2}\right]$. Like Heckman et al. (1989) we have calculated the physical beam area by using the distances given by heliocentric radial velocities. The $\int L_{\mathrm{CO}}{ }_{1 \rightarrow 0}$ refers to the global CO $1 \rightarrow 0$ luminosity $\left[\times 10^{3} \mathrm{~K} \mathrm{~km} \mathrm{~s}^{-1} \mathrm{kpc}^{2}\right]:{ }^{a}$ Young et al. (1995), ${ }^{b}$ Sandqvist et al. (1995) (over $\left.204^{\prime \prime} \times 164^{\prime \prime}\right)$ and ${ }^{c}$ Bryant \& Scoville (1996). $L_{\mathrm{FIR}}\left[10^{10} L_{\odot}\right]$ refers to the far infrared luminosity computed using the FIR flux (Lonsdale et al. 1985) and $v$ is the heliocentric radial velocity (NASA/IPAC Extragalactic Database). Adapted from Curran et al. (2000)

\begin{tabular}{lcccccr}
\hline Galaxy & Sy & $L_{\mathrm{CO} 1 \rightarrow 0}$ & $\int L_{\mathrm{CO} 1 \rightarrow 0}$ & $L_{\mathrm{HCN} 1 \rightarrow 0}$ & $L_{\mathrm{FIR}}$ & $v\left[\mathrm{~km} \mathrm{~s}^{-1}\right]$ \\
\hline NGC 0034 & 2 & $2.3 \pm 0.1$ & - & $0.56 \pm 0.07$ & 14.3 & 5931 \\
NGC 0931 & 1 & $0.08 \pm 0.04$ & - & $<0.1$ & 2.1 & 5001 \\
NGC 1068 & 2 & $0.42 \pm 0.01$ & $23 \pm 8^{a}$ & $0.09 \pm 0.01$ & 7.4 & 1134 \\
NGC 1365 & 2 & $1.5 \pm 0.1$ & $5.3^{b}$ & $0.16 \pm 0.03$ & 6.8 & 1636 \\
NGC 1667 & 2 & $1.18 \pm 0.08$ & - & $0.45 \pm 0.06$ & 4.2 & 4547 \\
UGC 03374 & 1 & $0.40 \pm 0.04$ & - & $<0.05$ & 2.9 & 6141 \\
NGC 2273 & 2 & $0.041 \pm 0.003$ & $0.38 \pm 0.07^{a}$ & $0.008 \pm 0.004$ & 0.66 & 1840 \\
Mrk 10 & 1 & $0.3 \pm 0.1$ & - & $<0.1$ & 2.7 & 8770 \\
NGC 4593 & 1 & $0.08 \pm 0.02$ & - & - & 0.79 & 2698 \\
Mrk 231 & 1 & $6.0 \pm 0.6$ & $5^{c}$ & $1.0 \pm 0.2$ & 128 & 12651 \\
NGC 5033 & 2 & $0.093 \pm 0.006$ & $10 \pm 3^{a}$ & $0.014 \pm 0.002$ & 0.53 & 875 \\
Mrk 273 & 2 & $2.4 \pm 0.4$ & - & $2.4 \pm 0.8$ & 73 & 11318 \\
NGC 5135 & 2 & $2.0 \pm 0.1$ & - & $0.11 \pm 0.01$ & 9.0 & 4112 \\
NGC 5347 & 2 & $0.03 \pm 0.01$ & - & $<0.01$ & 0.28 & 2336 \\
NGC 5548 & 1 & $0.4 \pm 0.2$ & - & - & 0.86 & 5149 \\
Arp 220 & 2 & $2.6 \pm 0.1$ & - & $0.4 \pm 0.2$ & 84 & 5314 \\
NGC 6814 & 1 & $0.118 \pm 0.002$ & $0.38 \pm 0.06^{a}$ & $0.011 \pm 0.002$ & 0.66 & 1563 \\
NGC 7130 & 2 & $2.6 \pm 0.2$ & - & $0.16 \pm 0.02$ & 11.9 & 4842 \\
NGC 7172 & 2 & $0.23 \pm 0.04$ & - & - & 1.2 & 2603 \\
NGC 7469 & 1 & $2.00 \pm 0.09$ & $1.9 \pm 0.3^{a}$ & $0.25 \pm 0.07$ & 18.2 & 4889 \\
\hline
\end{tabular}

(Table 1). If we exclude these from the sample, we find $L_{\mathrm{FIR}}(\mathrm{Sy} 2) \approx 1.4 L_{\mathrm{FIR}}(\mathrm{Sy} 1)$ and if we take the averages for all of the sample with $^{6} v \gtrsim 4000 \mathrm{~km} \mathrm{~s}^{-1}$ the factor remains similar (1.3). Applying this factor to Eq. (2) gives $L_{\mathrm{CO}}(\mathrm{Sy} 2) \approx 2 L_{\mathrm{CO}}(\mathrm{Sy} 1)$, i.e. the result of Heckman et al. (1989). When we examine their observational results, however, we find that the single position observations have been used to determine the luminosities for all of the observed sample. As mentioned in Fig. 1, we believe that the $\mathrm{CO}$ is only fully sampled ${ }^{7}$ in sources in which $v \gtrsim 4000 \mathrm{~km} \mathrm{~s}^{-1}$, or in the case of the NRAO $12 \mathrm{~m} 55^{\prime \prime}$ beam, $v \gtrsim 3500 \mathrm{~km} \mathrm{~s}^{-1}$, which leaves the same "distant" sample as ours. It is not quite clear whether their method (normalising the beam area to the optical area) takes this effect fully into account when calculating CO luminosities for the $v \lesssim 3500 \mathrm{~km} \mathrm{~s}^{-1}$ (near-by) sample. Note that we obtain the same result as Eq. (1) when we use the whole sample without considering beam-filling, i.e. using the third column of Table 1 for the CO luminosities regardless of $v$.

In the case of Maiolino et al. (1997), they have excluded sources in which the beam does not sample out to radii beyond $4 \mathrm{kpc}$, i.e. $v \lesssim 2300 \mathrm{~km} \mathrm{~s}^{-1}$ with the

\footnotetext{
6 Thus ruling out the bias.

7 For example, as observed in NGC 1068 (Planesas et al. 1989) and NGC 1365 Sandqvist et al. (1995).
}

NRAO $12 \mathrm{~m}$. Their sample corresponds to our distant sample, suggesting that $\frac{L_{\mathrm{CO}}}{L_{\mathrm{FIR}}}(\mathrm{Sy} 2) \approx \frac{L_{\mathrm{CO}}}{L_{\mathrm{FIR}}}(\mathrm{Sy} 1)$ for $v \gtrsim$ $4000 \mathrm{~km} \mathrm{~s}^{-1}$. However, Fig. 1, which uses the same sample, suggests that $\frac{L_{\mathrm{CO}}}{L_{\mathrm{FIR}}}(\mathrm{Sy} 2) \approx 3 \frac{L_{\mathrm{CO}}}{L_{\mathrm{FIR}}}(\mathrm{Sy} 1)$, which is also found to apply for the whole sample (according to the literature). Referring to Curran et al. (2000), we see that, asides from the differences in mean $L_{\mathrm{FIR}}$ between the nearby and distant galaxies, $L_{\mathrm{CO}} / L_{\mathrm{HCN}} \approx 6$ (distant Seyferts) cf. $L_{\mathrm{CO}} / L_{\mathrm{HCN}} \approx 17$ (all of the Seyferts) and so it appears as though there exists a distinct difference between the near-by and distant samples. This is discussed in Sect. 4.

\section{Differences in the aspect of the molecular gas distribution between type 1 and type 2 Seyferts}

In this section we employ a model of the molecular gas in order to try and determine the orientation of the molecular gas for each of the galaxies of Curran et al. (2000). The model, which is discussed in more detail in Curran (1998); Curran (2000), provides a very satisfactory fit to the observed data in the Circinus galaxy, a type 2 Seyfert $^{8}$,

\footnotetext{
${ }^{8}$ Regarding the previous section, note that Circinus, the closest lying example of a Seyfert galaxy, has $L_{\mathrm{FIR}}=6.210^{9} \mathrm{~L}_{\odot}$ (Lonsdale et al. 1985) and $L_{\mathrm{CO}} \approx 3010^{3} \mathrm{~K} \mathrm{~km} \mathrm{~s}^{-1} \mathrm{kpc}^{2}$ (Curran 2000), thus giving the high CO/FIR luminosity ratio
} 
which agrees closely with other observations of the gas in this galaxy (Veilleux \& Bland-Hawthorn 1997; Elmouttie et al. 1998a; Elmouttie et al. 1998b). Due to the close proximity of Circinus we could map the CO emission with the $22^{\prime \prime}$ beam at SEST, but since these galaxies are much further away, we have no such luxury (especially with the eight Southern galaxies $)^{9}$. However, since the optically thick molecular gas is unresolved (e.g. $\approx 13^{\prime \prime}$ in the relatively near-by NGC 1365 , Sandqvist 1999, cf. the $45^{\prime \prime}$ $\mathrm{HPBW}$ for $\mathrm{CO} 1 \rightarrow 0$ at SEST), the observed profiles will be a result of the gas distribution convolved over the velocity range. This technique has previously been used by Downes \& Solomon (1998) in Mrk 231. In Figs. 2 and 3 we show the unresolved central $10^{\prime \prime}$ of Circinus in the CO $2 \rightarrow 1$ and $1 \rightarrow 0$ transitions compared with the model of Curran et al. (1998).

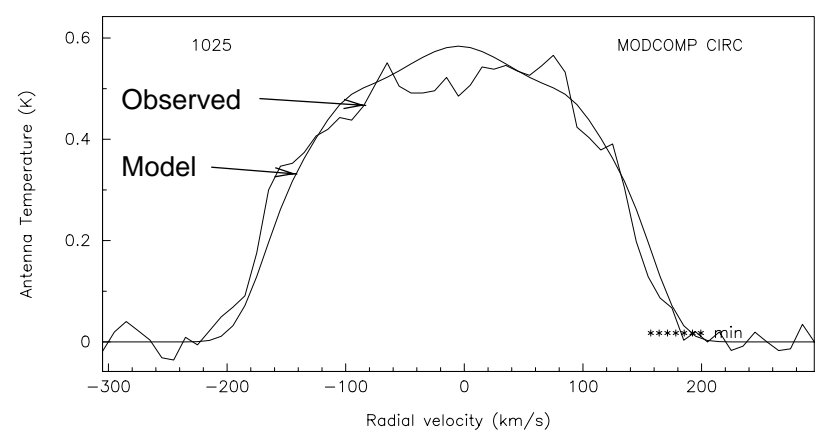

Fig. 2. The Circinus model "observed" with a $22^{\prime \prime}(\mathrm{CO} 2 \rightarrow 1$ at SEST) beam superimposed upon the CO $2 \rightarrow 1$ observed spectrum. Taken from Curran et al. (1998)

The molecular gas, which is assumed to be in a disc distribution, is modelled as:

1. The basic ring model of Curran et al. (1998);

2. The filled ring model in which the intensity at the inner edge of the ring is extrapolated to zero radius, while maintaining a velocity of $100 \mathrm{~km} \mathrm{~s}^{-1}$ (Sect. 3.2 .3 of Curran et al. 1998);

3. A disc constructed from the filled ring model but with the central velocity extrapolated to zero (Fig. 9 of Curran et al. 1998);

4. The ring+outflow model of Curran et al. (1999). In addition to an outflow perpendicular to the ring, we constructed versions in which an edge-on, a face-on and an outflow of intermediate orientation were added to rings of various inclinations.

These models were then tested at various inclinations and compared to the observed CO spectra (Fig. 4) in order to $\overline{\text { expected from the } L_{\mathrm{FIR}}} \sim 10^{10} L_{\odot}$ sample. Similar luminosities are found in the next furthest Seyfert NGC 4945 (Curran 2000).

${ }^{9}$ Where high resolution data is available, it is compared with our results.

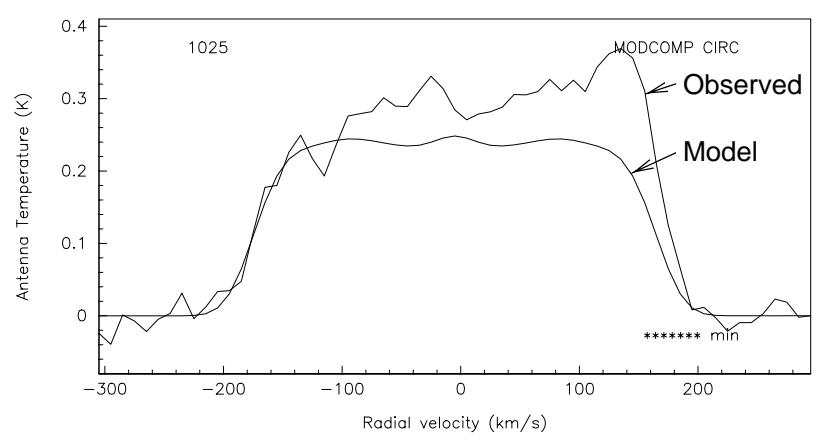

Fig. 3. The Circinus model observed with a $45^{\prime \prime}(\mathrm{CO} 1 \rightarrow 0$ at SEST) beam superimposed upon the $\mathrm{CO} 1 \rightarrow 0$ observed spectrum. Perhaps due to a small pointing error $\left(\lesssim 5^{\prime \prime}\right)$, the observed spectrum is not as symmetrical as that of CO $2 \rightarrow 1$ (Fig. 2), although it is seen that the model still provides a good match

see if any obvious matches could be found. This was done for the sources in which a clean profile of the $\mathrm{CO}$ was obtained; we have excluded the relatively poor NGC 0931, and Mrk 10 detections (Curran et al. 2000). By obvious matches we mean that it was our priority to try to reproduce the observed profile shapes before trying to match the integrated intensities (i.e. velocity widths and antenna temperatures). For this purpose matching the model and observed profile by eye was sufficient since we didn't really expect to constrain the inclination to closer than $\approx 20^{\circ}$ accuracy, and as seen from Fig. 5, differences in the inclination are easily discernable. Naturally some profiles are not unique, e.g. for NGC 5135 (Sect. 3.11), where a disc or a ring model may apply. The model was good enough, however, to give an approximate inclination for a particular model and any variations on the disc distribution which fitted the observed profile have also been noted. If reasonable matches were found, the models were then "observed" with the required HPBW in order to simulate the model at different apparent sizes. As previously (Curran et al. 1999), we (initially) scaled the beam according to optical major axis of the galaxy rather than the distance (velocity $)^{10}$. This method assumes that the extent of the ring/disc molecular gas distribution is proportional to the size of the galaxy as opposed to being the same size for all of the sample (i.e. a radius of $\sim 600$ pc, Curran et al. 1998). As long as we are not simulating near-by systems, where the beam is filled, Curran et al. 1999), the choice of beam size will only affect the model intensity and not the profile shape. Examples of model profiles are shown in Fig. 5, and a more extensive range of these is shown in Curran (2000).

$\overline{10}$ Throughout this paper we assume $H_{0}=75 \mathrm{~km} \mathrm{~s}^{-1} \mathrm{Mpc}^{-1}$. 

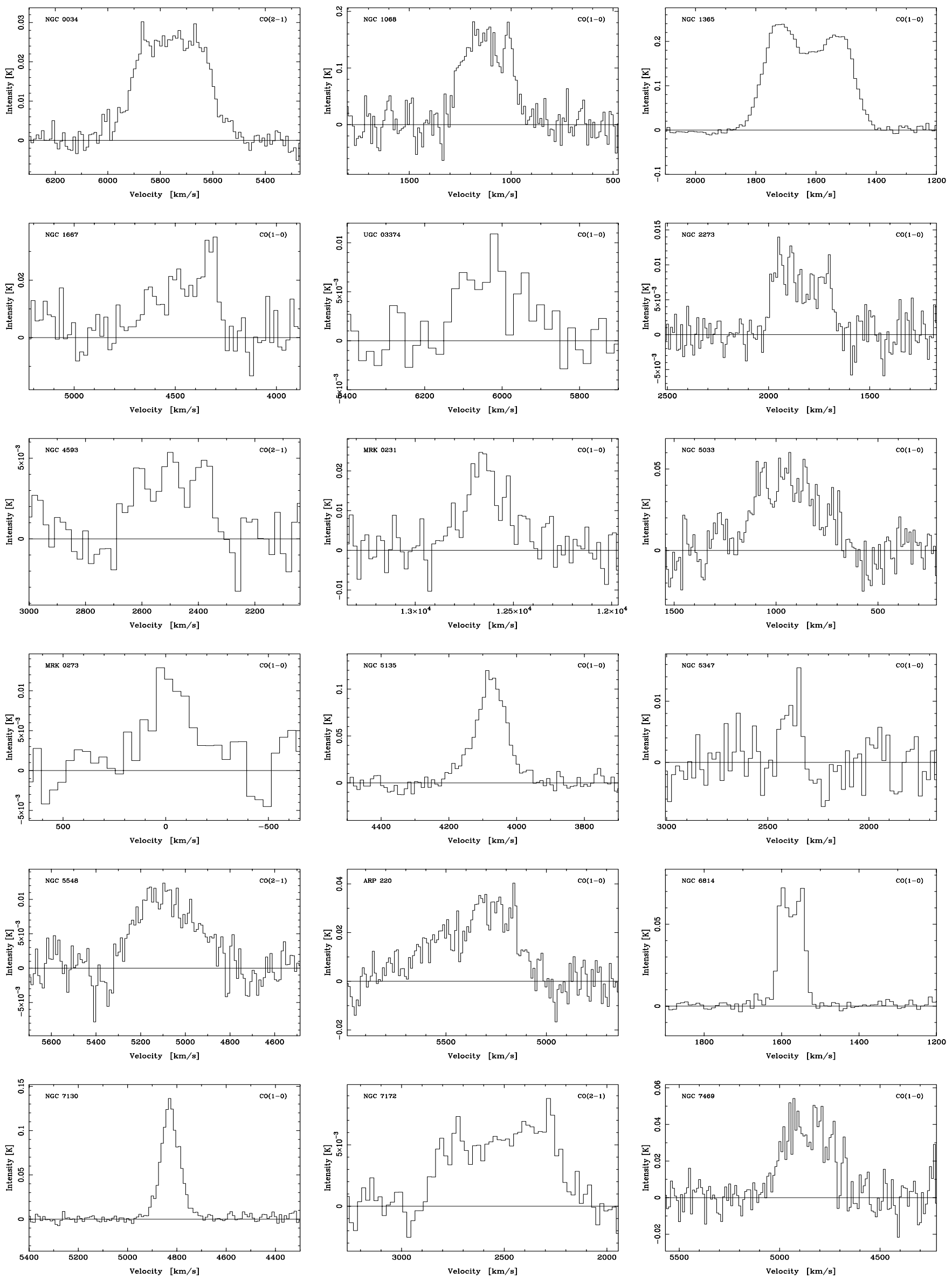

Fig. 4. The observed spectra of Curran et al. (2000) shown to a velocity resolution of $10 \mathrm{~km} \mathrm{~s}^{-1}$, except NGC 0034, NGC 1667, UGC 03374, NGC 4593, Mrk 231, NGCs 5347 and 7172 to $20 \mathrm{~km} \mathrm{~s}^{-1}$ and Mrk 273 (shown relative to $z=0.037780$ ) to $40 \mathrm{~km} \mathrm{~s}^{-1}$. The intensity scale is $T_{\mathrm{A}}^{*}$ and the velocity scale is relative to the local standard of rest (l.s.r.) 

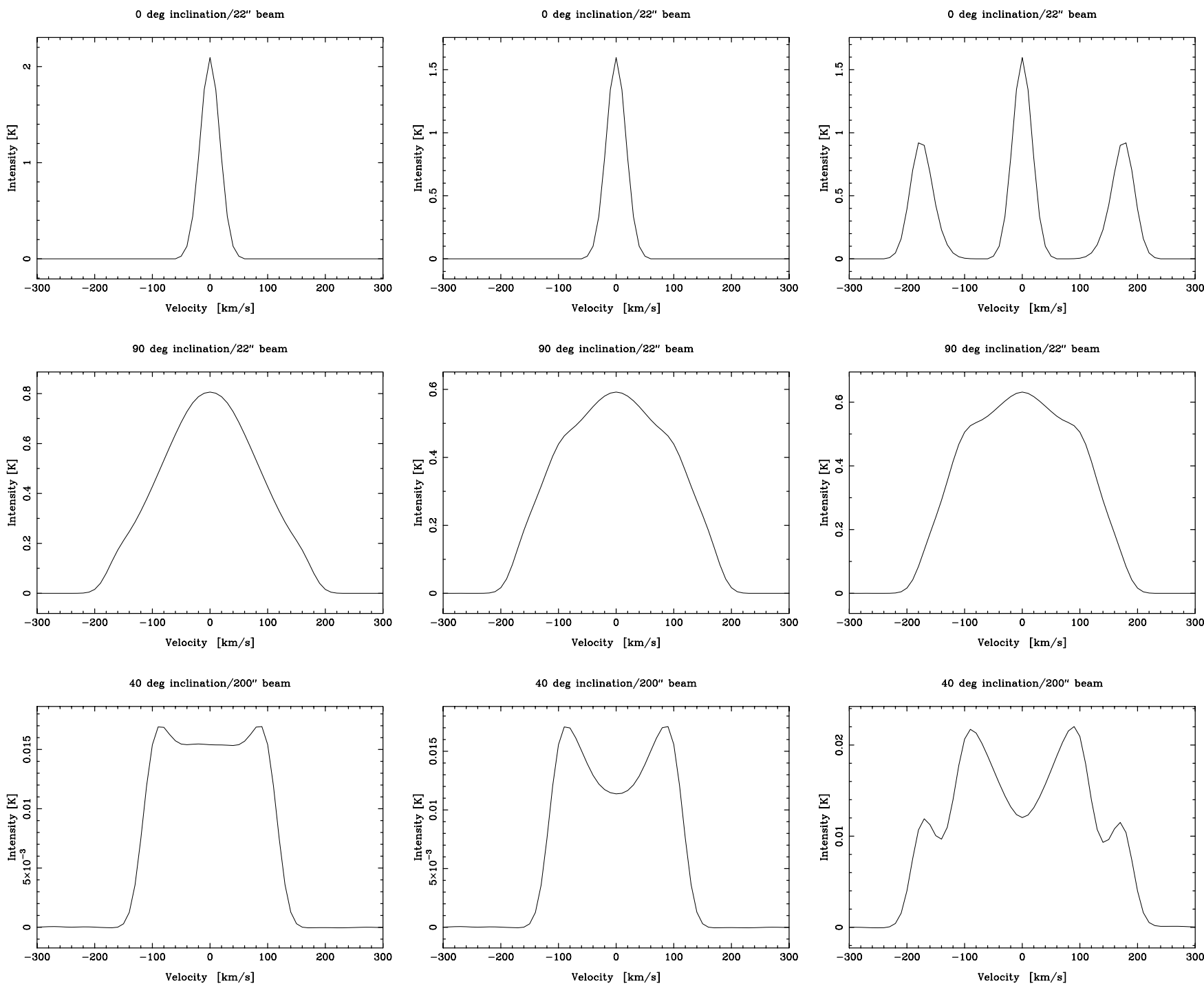

Fig. 5. Examples of various models (further examples are shown in Curran 2000). Left; a disc model, centre; a ring model and right; a ring+(perpendicular) outflow model. The model inclinations and HPBWs used to "observe" them are shown in each case; a $22^{\prime \prime}$ beam corresponds to Circinus for CO $2 \rightarrow 1$ at SEST

\section{1. $N G C 0034$}

The observed $2 \rightarrow 1$ profile of NGC 0034 generally resembles that of a highly inclined disc/ring model, but with "ears" at around 5700 and $5900 \mathrm{~km} \mathrm{~s}^{-1}$. These in conjuction with the central hump gives the observed spectrum a slightly 3-peaked profile, and its presence is consistent with a ring+outflow model at inclinations of $\approx 60^{\circ}$. The outflow angle is more difficult to constrain, although we can say that the outflow has an inclination of between $0^{\circ}$ (edge-on) and $60^{\circ}$ (normal to the ring), Fig. 6.

To summarise, since a ring+outflow gives such a 3peaked profile (the ring and filled-ring models give a 2peaked profile), we suggest that the gas may be distributed in such a fashion, although, due to the model limits and the noisier $1 \rightarrow 0$ spectrum, all we can say with some confidence is that the gas appears to be in a ring of fairly high inclination $\left(\gtrsim 60^{\circ}\right)$. It should be noted that a beam-width of $70^{\prime \prime}$, which is scaled for the optical disc, gives an intensity which is $\approx 5$ times the observed. In order to achieve the same intensity, the model has to be "observed" with a 175 " beam, Fig. 6 . Since, assuming a similar small-scale filling to the gas in Circinus, a $\left(\frac{79 \mathrm{Mpc}}{4 \mathrm{Mpc}}\right) \times 22^{\prime \prime} \approx 430^{\prime \prime}$ beam would be required to scale NGC 0034 according to the distance ${ }^{11}$, we conclude that the gas extends $430^{\prime \prime} / 175^{\prime \prime}=2.5$ times as far as in Circinus $^{12}$, i.e. to $\approx 1.5 \mathrm{kpc}$.

\footnotetext{
${ }_{11}$ Since we compare beam sizes it is not necessary to take into account the main beam efficiencies.

12 The luminosity (Curran et al. 2000) could also contribute to the observed intensity, cf. Circinus (Curran et al. 1998; Curran 2000), and so we assign limits only, Table 2.
} 


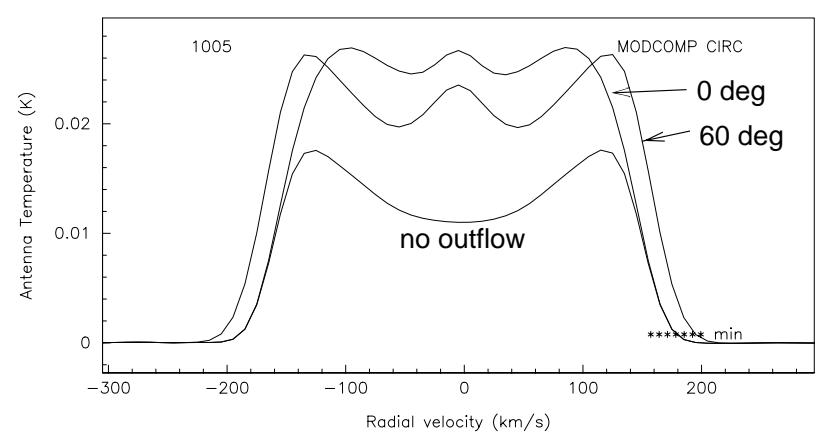

Fig. 6. A $60^{\circ}$ inclination ring(+outflow) "observed" with a $175^{\prime \prime}$ beam. The labels refer to the outflow inclination

\section{2. $N G C 1068$}

The width of the $1 \rightarrow 0$ line is suggestive of an inclined disc/ring system with the sheer of the profile edges favouring the (filled or unfilled) ring model ${ }^{13}$. Although the profile is too complex to make anything but broad estimates, the profile shape and width suggest that ring has an intermediate inclination $\left(40^{\circ}-60^{\circ}\right)$ with the possibility of a nearly edge-on outflow. This is expected to be inclined close to the ionisation cone (Wilson \& Tsvetanov 1994; Curran et al. 1999), which should be close to edge-on in a Sy2 nucleus (Antonucci \& Miller 1985; Wilson et al. 1988; Tadhunter \& Tsvetanov 1989; Wilson \& Tsvetanov 1994; Baker \& Scoville 1998). Also, although in this case the model is not so certain, the observed intensity is obtained when it is "observed" with $\mathrm{a} \approx 70^{\prime \prime}$ beam. This is twice the width of a beam scaled for the optical extent. Since based upon distance, a beam width of $\approx 140^{\prime \prime}$ is required to observe the model at $15 \mathrm{Mpc}$, we can estimate the extent of the molecular gas as being twice that in Circinus, i.e. $\approx 1.3 \mathrm{kpc}$. Thus the model gives similar results to the interferometric results of Tacconi et al. (1994); Papadopoulos (1996) who determine molecular disc inclinations of $30-50^{\circ 14}$ and $\approx 50^{\circ}$ (extending to $\approx 1.8 \mathrm{kpc}$ ), respectively.

\section{3. $N G C 1365$}

As seen from Fig. 7, the Circinus (filled and unfilled) ring model gives a very similarly shaped profile to that of the observed $^{15}$ for inclinations of $30^{\circ}$ to $40^{\circ}$. In the case of

\footnotetext{
13 A disc model gives a gentler rise.

14 Although they do narrow this range down to $30-40^{\circ}$ from their models.

15 Applying a $14^{\prime \prime}$ beam to the model and comparing it with the corresponding $2 \rightarrow 1$ spectrum, we find that not such a good match is achieved (cf. Fig. 7) between the model profile and that in Fig. 4. For the $2 \rightarrow 1$ transition the model spectrum is similar in shape to that in Fig. 7 and with an intensity of $0.47 \mathrm{~K}$ (cf. $T_{\mathrm{mb}} \approx 0.5 \mathrm{~K}$, Curran et al. 2000 ) while the ob-
}

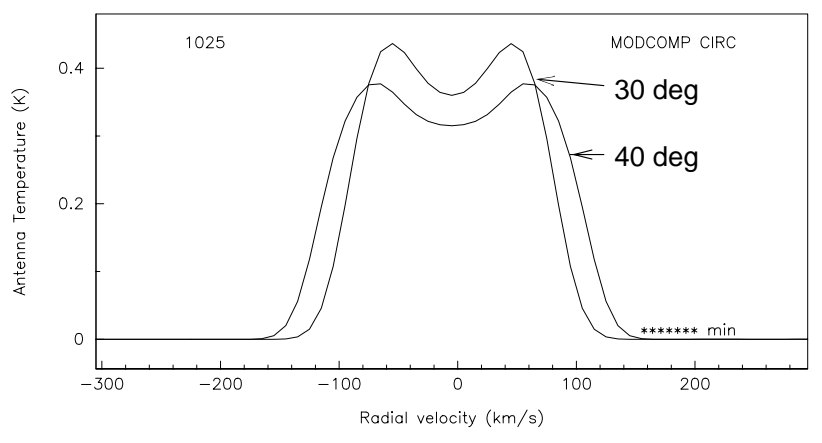

Fig. 7. The ring only model at $30^{\circ}$ and $40^{\circ}$ inclinations observed with a $28^{\prime \prime}$ beam, i.e. NGC 1365 scaled for the apparent optical size when observed in CO $1 \rightarrow 0$ at SEST

the model, however, the intensity is similar ${ }^{16}$, which suggests that the extent of the gas is nearly proportional to the optical disc size, i.e. nearly 5 times as extended as in Circinus $^{17}$ (to a radius of $\lesssim 3 \mathrm{kpc}$ ). From VLA and SEST observations, Sandqvist et al. (1995) find the molecular gas to lie within the central $2.2 \mathrm{kpc}$.

A significant difference, however, is the fact that the model profile is narrower than the observed by a factor of $\approx 2$. This suggests that the maximum de-projected velocity in the ring of NGC 1365 is double that in Circinus i.e. $\approx \pm 400 \mathrm{~km} \mathrm{~s}^{-1}$ (Curran et al. 1998). Since $M \propto v^{2} r$, this implies that the molecular gas mass (assuming a relatively small central dynamical mass, e.g. $10 \%$ of the gas mass, Curran 1998) is (at least) four times that in Circinus. Interestingly, Sandqvist et al. (1995) find the molecular gas mass in the central region of NGC 1365 to be $6.310^{9} M_{\odot}$, i.e. also 4 times that in Circinus (Curran et al. 1998 $)^{18}$. It is possible, however, that since the extent of the gas in NGC 1365 may be considerably larger, this factor $^{19}$ would require an even larger gas mass/different physical conditions to those in Circinus.

Like Circinus (Marconi et al. 1994; Veilleux \& BlandHawthorn 1997; Elmouttie et al. 1998b), NGC 1365 exhibits an ionisation cone Hjelm \& Lindblad 1996) and since the model cannot distinguish the presence of an outflow of low inclination, we cannot exclude the presence of this (Curran 2000). It should be noted, however, that the

served is quite asymmetric. We believe that this is due to a pointing error (Curran et al. 2000).

${ }^{16} \mathrm{In}$ fact the same for $\eta_{\mathrm{mb}}=0.7$ at SEST, giving $T_{\mathrm{mb}}=$ $0.36 \mathrm{~K}$ for an inclination of $40^{\circ}$.

17 NGC 1365 is approximately five times the distance of Circinus and so based on this, a $220^{\prime \prime}$ beam would be required to simulate a $600 \mathrm{pc}$ ring, although a $40^{\prime \prime}$ beam (approximately the $1 \rightarrow 0$ beam at SEST) gives the observed intensity.

18 Using the $N_{\mathrm{H}_{2}} / L_{\mathrm{CO}}$ Galactic conversion ratio in both cases (Strong et al. 1988).

19 Although $\approx 600 \mathrm{pc}$ is just the bulk ring/disc extent in Circinus. The CO $1 \rightarrow 0$ transition has been detected out to nearly $4 \mathrm{kpc}$ along the major axis (Curran et al. 2000a). 
observed profile may arise from the presence of the strong bar in NGC 1365 (e.g. Teuben et al. 1986; Lindblad et al. 1996), although, as yet, current observations (e.g. Sandqvist et al. 1995) are of insufficient resolution in order to distinguish whether the $\mathrm{CO}$ distribution expected from the bar is present (Kenney et al. 1992; Kenney 1996). In summary, all we can say is that, if a molecular ring is the dominant component on the sub-kpc scale $^{20}$, then our model suggests that it has an inclination of between $30^{\circ}$ and $40^{\circ}$ which puts it coplanar to the obscuring torus and large scale galactic disc (Wilson \& Tsvetanov 1994; Hjelm \& Lindblad 1996).

\subsection{NGC $166^{r}$}

Since the detection of NGC 1667 may have been compromised by a pointing error, Fig. 4, we assume that this has a symmetric form as in Papadopoulos \& Seaquist (1998). Although gentler, the profile has a similar shape to that of NGC 1365 . When we observe ring models at inclinations of $\approx 30^{\circ}$ with a $125^{\prime \prime}$ beam $(\mathrm{CO} 1 \rightarrow 0$ at $\mathrm{OSO})$ we obtain a similar general shape and intensity, although the velocity range is again about a factor of 2 too small. Since Circinus has a similar CO luminosity within 800 pc as NGC 1667 out to $\approx 5 \mathrm{kpc}\left(\approx 110^{3} \mathrm{~K} \mathrm{~km} \mathrm{~s}^{-1} \mathrm{kpc}^{2}\right.$, Curran et al. 1998; Curran et al. 2000), a smaller extent in the molecular ring/disc could account for the higher velocities. Of particular interest, however, is the fact that the addition of a (strong, close to edge-on) molecular outflow is required in order to produce the observed central hump (Fig. 4 and Maiolino et al. 1997; Papadopoulos \& Seaquist 1998).

\section{5. $U G C 03374$}

The low signal-to-noise $(\mathrm{S} / \mathrm{N})$ ratio in this source does not allow us to be quite as specific when fitting a model, but the observed spectrum (Fig. 4 and Maiolino et al. 1997) may be fitted by a disc ${ }^{21}$ of any inclination or a ring of inclination $\lesssim 20^{\circ}$. In order to account for the observed intensity, a beam width exceeding $200^{\prime \prime}$ (the limit of the model program) is required, although the lower luminosity $\left(0.410^{3} \mathrm{~K} \mathrm{~km} \mathrm{~s}^{-1} \mathrm{kpc}^{2}\right.$, Curran et al. 2000) could contribute to this.

\footnotetext{
20 A fast rotating disc is believed to be present within the central $~ 700$ pc (Burbridge \& Burbridge 1960; Lindblad 1978), and Sandqvist (1999) has recently observed $\mathrm{a} \approx 600 \mathrm{pc}$ molecular ring in $\mathrm{CO} 3 \rightarrow 2$. In the case of the $1 \rightarrow 0$ transition, we would expect the molecular gas to be more extended than this; by perhaps a factor of $\approx 2$, e.g. as in the Sy2s Circinus and NGC 4945 (Dahlem et al. 1993; Mauersberger et al. 1996; Curran et al. 1998; Curran et al. 2000b).

${ }^{21}$ For the disc model an inclination of $\gtrsim 40^{\circ}$ gives a comparable velocity width to the ring model.
}

\section{6. $N G C 2273$}

Again the $\mathrm{S} / \mathrm{N}$ ratio is too low in order to distinguish particular details (i.e. the presence of an outflow), although the general shape does suggest a (filled or unfilled) ring rather than a disc. From the shape of the profile, we estimate this ring to have an inclination of between $20^{\circ}$ and $50^{\circ}$. Again, in order to account for the observed intensity, a beam width exceeding $200^{\prime \prime}$ is required. This is the size of beam required to scale NGC 2273 according to distance, and so we suggest that the molecular gas is more confined than the ring/disc in Circinus, although again the lower CO luminosity $\left(0.410^{3} \mathrm{~K} \mathrm{~km} \mathrm{~s}^{-1} \mathrm{kpc}^{2}\right.$, Young et al. 1995) may affect this.

\section{7. $N G C 4593$}

Again in the case of the $\mathrm{CO} 1 \rightarrow 0$ profile, the $\mathrm{S} / \mathrm{N}$ ratio is too low to distinguish any details, although the shape of the spectrum does suggest a close to edge-on ring or disc, thus orientating the gas close to perpendicular to the torus of the Sy1 nucleus. Looking at the CO $2 \rightarrow 1$ profile (Fig. 4), we see, however, that the width may be due to a 3 component system. Such a profile is what we might expect if a close to face-on ring+outflow were present (Curran et al. 1999). With a face-on (Sy1) outflow, the observed spectrum is best fitted with a ring of $\lesssim 20^{\circ}$ inclination, Fig. 8.

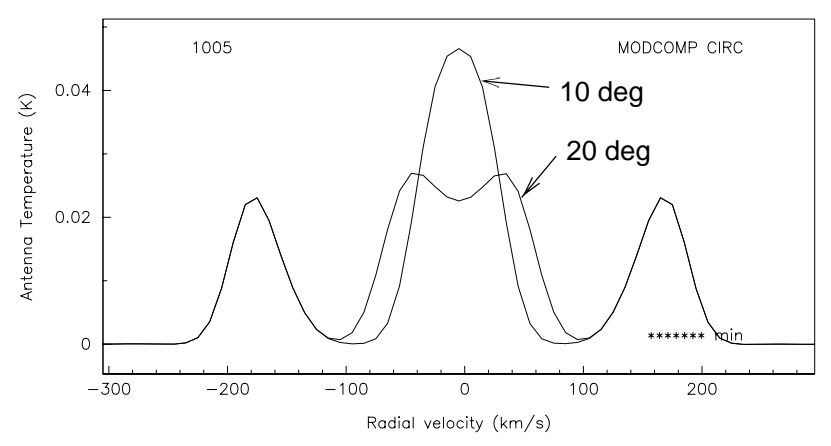

Fig. 8. The ring (at inclinations of $10^{\circ}$ and $20^{\circ}$ ) +outflow (close to face-on) model observed with a $200^{\prime \prime}$ beam, i.e. scaled for a $600 \mathrm{pc}$ ring at $39 \mathrm{Mpc}$. The fact that this is still $\approx 10$ times the observed intensity suggests that the molecular gas extent is much less than in Circinus. The optical disc scale (39" beam for CO $2 \rightarrow 1$ at SEST) gives an intensity of over 10 times that shown (i.e. 100 times the observed). In the figure the outflow features $\left(\approx \pm 180 \mathrm{~km} \mathrm{~s}^{-1}\right)$ overlap for both ring inclinations

One difference between the model and the observed spectrum is the fact that the peripheral (model outflow) features occur at $\approx \pm 90 \mathrm{~km} \mathrm{~s}^{-1}$ in the observed spectrum. This "slower outflow", cf. Circinus, may be due to the fact that NGC 4593 is considerably less luminous 
(Curran et al. 2000) and this would also explain the low observed intensity, in addition/as an alternative to a confined molecular gas distribution, Fig. 8.

\subsection{Mrk 231}

Although the $\mathrm{S} / \mathrm{N}$ ratio is significantly poorer, like NGC 5135 (Sect. 3.11) the observed $1 \rightarrow 0$ profile of Mrk 231 could be due to either a disc or ring distribution at fairly low $\left(\lesssim 30^{\circ}\right)$ inclinations ${ }^{22}$. Note that Downes \& Solomon (1998) find a nearly face-on molecular disc from their model. From the intensity of the observed profile we estimate an extent of $\mathrm{CO}$ emission which is about 7 times $^{23}$ that in Circinus, i.e. out to $\sim 4 \mathrm{kpc}$. This is an order of magnitude higher than the radius determined from the interferometric measurements of Bryant \& Scoville (1996), and in this case we must therefore conclude that the relatively high observed intensity is a result of the high intrinsic CO luminosity in Mrk 231 ( $\lesssim 5$ times Circinus within the beam, Curran et al. 1998; Curran et al. 2000) rather than due to a large extent. This emphasises that any values we obtain for the CO extent, should be considered as limits only, Table 2.

\section{9. $N G C 5033$}

As with CO $2 \rightarrow 1$ in NGC 0034, the spectra of Maiolino et al. (1997); Papadopoulos \& Seaquist (1998) suggest a (filled or unfilled) ring, possibly with an outflow, which has a fairly high $\left(\gtrsim 60^{\circ}\right)$ inclination, although this is not so apparent in our noisy $1 \rightarrow 0$ detection, Fig. 4 . The beam size used to obtain the observed intensity is based upon distance. Although as in the case of Mrk 231 (Sect. 3.8), this may be a consequence of the high intrinsic luminosity (Young et al. 1995).

\subsection{Mrk 273}

In Mrk 273 the $\mathrm{S} / \mathrm{N}$ ratio is too poor in order to assign a model. From the intensity, however, we can estimate that a HPBW of $400^{\prime \prime}$ produces the observed intensity, as opposed to the $1200^{\prime \prime}$ beam expected if the molecular gas in Circinus were scaled for distance. This leads us to conclude that the molecular gas extends to around ${ }^{24} \sim 2 \mathrm{kpc}$. This is comparable with the $\approx 2.5 \mathrm{kpc}$ obtained from the

\footnotetext{
22 Bryant \& Scoville (1996) estimate an inclination of $\lesssim$ $22^{\circ}$ providing the $N_{\mathrm{H}_{2}} / L_{\mathrm{CO}}$ Galactic conversion applies, although this is questionable in ultra-luminous/Seyfert galaxies (Maloney \& Black 1988; Maloney 1990; Shier et al. 1994; Mauersberger et al. 1996; Curran et al. 1998).

23 A $200^{\prime \prime}$ beam gives the observed intensity as opposed to the $1400^{\prime \prime}$ beam expected from the distance scale.

${ }^{24}$ Since the relatively high luminosity within the beam (7 times Circinus) may lead to an overestimate (Sect. 3.8).
}

interferometric map of Yun \& Scoville (1995). Assuming a flat circular distribution, the disc has an inclination of $\approx 60^{\circ}$, which we shall adopt.

\subsection{1. $N G C 5135$}

Both the $1 \rightarrow 0$ and CO $2 \rightarrow 1$ observed spectra can only be fitted with disc/ring model of low inclination, although this produces a somewhat narrow profile (Fig. 9). As before (Sect. 3.4), a more confined gas could account for the high velocities, although the addition of an outflow could achieve this, Fig. 9. A sufficiently high $\mathrm{S} / \mathrm{N}$ ratio for the profile wings would be required in order to see this in the observed profile, Fig. 4.

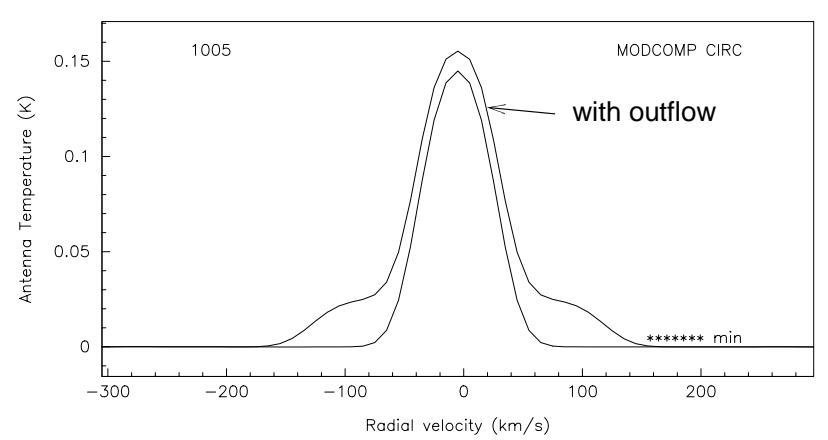

Fig. 9. The disc and ring $\left(10^{\circ}\right)+$ outflow (edge-on) model observed with a $122^{\prime \prime}$ beam, i.e. scaled for NGC 5135 observed in $\mathrm{CO} 1 \rightarrow 0$ at SEST. The fact that the model intensity is similar to that of the observed, suggests that the molecular gas does scale with the optical disc for this luminous galaxy. Since we would require a $630^{\prime \prime}$ beam to place NGC 5135 at the same distance as Circinus we estimate that the ring/disc molecular gas is approximately 5 times as extended. This contradicts the fact that the profile width suggests a more confined gas and so we attribute the observed width to the higher intrinsic luminosity of NGC 5135 within the beam (Curran et al. 2000)

\subsection{2. $N G C 5347$}

When fitting the model, the narrow profile of this source is reminiscent of a lowly inclined disc or ring system (of $\sim 600 \mathrm{pc}$ extent). It should be noted, though, that NGC 5347 has a much lower luminosity than Circinus and so a dimmer (i.e. slower) system of higher inclination is possible.

\subsection{3. $N G C 5548$}

The $2 \rightarrow 1$ (and possibly $1 \rightarrow 0$ ) detection of this is reminiscent of an inclined $\left(\approx 70^{\circ}\right)$ disc or ring, although it is difficult to obtain a close fit from the model. 


\subsection{4. $\operatorname{Arp} 220$}

Again, we experienced difficulties in fitting the model to this spectrum; we could either account for the intensity and the width, but not the shape with a highly inclined disc, or account for the intensity and shape but not the width, with a disc of intermediate inclination. In any case, since $\mathrm{a} \sim 200^{\prime \prime}$ beam gives the observed intensity, it is feasible that the molecular ring/disc extends to around $2 \mathrm{kpc}$. Since the luminosity is slightly greater within the beam than of the ring/disc in Circinus $\left(2.610^{3} \mathrm{~K} \mathrm{~km} \mathrm{~s}^{-1} \mathrm{kpc}^{2}\right.$, Curran et al. 2000 $)^{25}$ a slightly larger beam would be required to obtain a match, thus decreasing this distance estimate slightly. From their interferometric observations, Scoville et al. (1997) find that the CO, which is distributed in a thin disc, extends out to $\approx 1.4 \mathrm{kpc}$. An inclination of $40^{\circ}-50^{\circ}$ is derived for the disc and so we adopt this value.

\subsection{NGC 6814}

The best match was obtained using a low inclination $\left(\lesssim 20^{\circ}\right)$ ring "observed" with a $104^{\prime \prime}$ beam, giving a profile similar to that in Fig. 5, although somewhat narrower. This suggests that the extent of the gas does scale as the optical extent in this case, although due to the proximity of this galaxy, this only corresponds to a radius of $\approx 1 \mathrm{kpc}$. It should be noted that the CO luminosity within the beam is somewhat lower than that in Circinus $\left(0.3810^{3} \mathrm{~K}\right.$ $\mathrm{km} \mathrm{s}^{-1} \mathrm{kpc}^{2}$, Young et al. 1995) which would cause a lower observed intensity and so this radius should be regarded as an upper limit only.

\subsection{NGC 7130}

Since the $1 \rightarrow 0$ and $2 \rightarrow 1$ observed spectra observed spectra are so narrow $\left(\lesssim \pm 100 \mathrm{~km} \mathrm{~s}^{-1}\right.$ ), we expect this disc structure to be of low inclination, and the profile shape is indeed best fitted with a close to face-on disc/ring observed with a $160^{\prime \prime}$ beam (as opposed to a $\approx 700^{\prime \prime}$ beam required for a $600 \mathrm{pc}$ ring at $64 \mathrm{Mpc}$ ), Fig. 10. This suggests that the ring/disc of molecular gas extends to around 4 times the distance than in Circinus. The luminosity within the beam is slightly greater than that in Circinus (2.6 $10^{3} \mathrm{~K} \mathrm{~km} \mathrm{~s}^{-1} \mathrm{kpc}^{2}$, Curran et al. 2000) and so a smaller radius may be required in to produce the observed velocity range. This range could feasibly be accounted for with a disc model of higher inclination but the model profile loses its "pointed" shape.

\footnotetext{
25 As previously mentioned, preliminary results from large scale observations of Circinus suggest a global CO luminosity of $\approx 3010^{3} \mathrm{~K} \mathrm{~km} \mathrm{~s}^{-1} \mathrm{kpc}^{2}$ out to $\approx 5 \mathrm{kpc}$ (Curran et al. 2000). It should be noted, however, that we are using a model of the molecular ring in Circinus, which has a luminosity of $\approx 5 \%$ of this, to model the rings/discs in these galaxies, in which the molecular gas is expected to be very centralised (Curran et al. 2000).
}

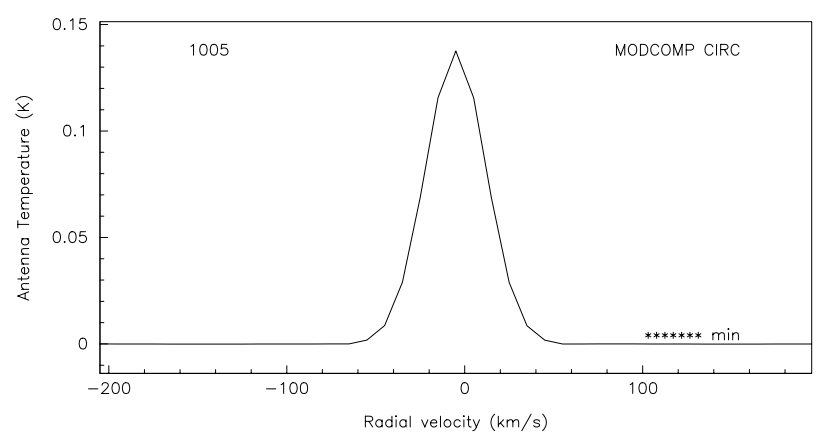

Fig. 10. A face-on disc observed with a $160^{\prime \prime}$ beam

\subsection{7. $N G C 7172$}

The $2 \rightarrow 1$ observed profile has a shape somewhat similar to that of NGC 0034, with the 3-component CO $1 \rightarrow 0$ shape of Maiolino et al. (1997) also suggesting an inclined ring, possibly with associated outflow. Testing various models, a $60^{\circ}$ ring+outflow model "observed" with a beam exceeding $200^{\prime \prime}$ gives the best match. Worth noting is that a ring only model (also of $60^{\circ}$ inclination) at a HPBW of $200^{\prime \prime}$ gives the observed intensity, although the central component in the profile is missing. So we summarise the model as being a highly inclined ring in which the $\mathrm{CO}$ is confined to $\lesssim 600 \mathrm{pc}$ with a possible outflow. The presence of the outflow in the model permits an extent of $\approx 600 \mathrm{pc}$ for the gas. The low intrinsic luminosity (0.23 $10^{3} \mathrm{~K} \mathrm{~km} \mathrm{~s}^{-1} \mathrm{kpc}^{2}$, Curran et al. 2000) could increase the values of the inferred radii.

\subsection{8. $N G C 7469$}

Although a 2-peaked profile may be feasible in our observed spectrum, and also in that of Heckman et al. (1989), no such feature is apparent in the detections of Maiolino et al. (1997); Papadopoulos \& Seaquist (1998). In fact we find that the best fit is provided by a ring/disc model at low inclinations, without the presence of an outflow. Also, our models show that a nearly-face on disc/ring gives the observed profile, but not the required width. A slightly more inclined disc (a ring does not suffice here) gives the required width, although the shape is perhaps only consistent with the detection of Papadopoulos \& Seaquist (1998), and the model should be "observed" with a smaller beam in order to obtain the observed intensity. Since NGC 7469 has a higher CO luminosity in the beam than Circinus $\left(2.010^{3} \mathrm{~K} \mathrm{~km} \mathrm{~s}^{-1} \mathrm{kpc}^{2}\right.$, Young et al. 1995; Curran et al. 2000), which could widen the profile slightly, we favour the narrower $\left(10^{\circ}\right.$ inclination $)$ model. Being a Sy1.2, this would align the ring close to the obscuration and the beam size suggests that the molecular gas extent does (nearly) scale with the optical disc in this case (i.e. about three times more extended than that in Circinus). 
Heckman et al. (1986); Wilson et al. (1991) derive 1 - few $\mathrm{kpc}$ for the radius of the star-burst ring.

\subsection{Discussion of the model results}

We have simulated the observed profiles using a model based on that of the Circinus ring. It should be noted, however, that the exact profile shapes depend upon the distribution of the gas. For example, because of the flat intensity distribution out to $\approx 300 \mathrm{pc}$, the model (with no outflow) distribution (Figs. 9 and 10 of Curran et al. 1998) will give a single peaked profile for an inclined disc/ring, Fig. 5. If the beam is significantly larger than this, however, a relative deficit in the low velocity gas and the large negative gradient in the intensity at high velocities (from $\approx 300 \mathrm{pc}$ to $\approx 400 \mathrm{pc}$ ) will give a double peaked profile, also shown in Fig. 5. This could feasibly account for the observed profiles for NGCs 0034, 2273 and 7172. Also, although the choice of rotation curve is not crucial to the profile shape (Curran 1998) ${ }^{26}$, a constantly rising rotation curve may possibly reproduce the observed spectrum of NGC 5548. Thus we emphasise again that these models can only provide a very rough indicator of the molecular gas distribution. Therefore in the previous discussion (Sects. 3.1-3.18) particular attention should only be paid to the derived inclinations, which define the profile shapes. These are summarised in Table 2 , and the values should only be considered as approximate estimates, although where interferometric data is avaliable, i.e. NGC $1068^{27}$ and Mrk 231, our estimates give comparable results (due to uncertainties in Mrk 273 and Arp 220, we have adopted inclinations from the literature, Table 2).

Addressing the issue of differences in orientation between the galactic disc, large-scale molecular ring and small-scale torus $^{28}$, Circinus, NGCs 0034, 1365, 5033, 7172 and Mrk 273 (which are all Sy2s) all have $i_{\text {main }} \approx$ $i_{\text {mol }} \approx$ dusty torus inclination (based on Seyfert type), which is consistent with the results of McLeod \& Rieke (1995); Wilson \& Tsvetanov (1994); Capetti et al. (1996). Examining this in more detail, the upper and lower limits in the inclination angles (Table 2) makes it difficult to select a median value, although for $i_{\text {main }}-i_{\text {mol }}$ this seems

\footnotetext{
${ }^{26}$ Assuming that the rotation curve does not deviate significantly from that of the ring in Circinus, e.g. a Keplerian curve (Curran et al. 1998; Curran 1998). A similar rotation curve was used by Downes \& Solomon (1998) to model the molecular gas as a disc in several ultra-luminous galaxies.

${ }^{27}$ Although our results only agree (at an inclination of $40^{\circ}$ ) with the favoured models of Tacconi et al. (1994), where streaming motions and disc warping are taken into account.

28 This is assuming that it is physically meaningful to distinguish different gas structures. Bands of various gas states do appear to be present, as molecular rings are observed in many Seyfert galaxies (Sect. 1), within which the atomic gas recombines under favourable conditions to form a masing/obscuring body (e.g. Neufeld \& Maloney 1995).
}

Table 2. Summary of the model results. $r$ is the estimated upper limit to the molecular gas extent in relation to Circinus (ring/disc to $\approx 600 \mathrm{pc}$ ). $i_{\mathrm{mol}}$ is the inclination of the molecular ring estimated from the models with the Circinus value coming from Curran et al. (1998). $i_{\text {main }}$ is the approximate inclination of the main galaxy disc. In most cases these have been calculated (assuming a circular shape) from the ratio of the major and minor optical axes (NASA/IPAC Extragalactic Database); the exceptions are Circinus (Freeman et al. 1977), NGC 1068 (Tacconi et al. 1994) and NGC 1365 (Hjelm \& Lindblad 1996), of which only the latter disagrees with the NASA/IPAC estimated value $\left(\approx 60^{\circ}\right)$. Again, Sy is the Seyfert type which is expected to be directly related to the inclination of the obscuration; Sy1 face-on, Sy2 edge-on and intermediate classes of intermediate inclinations. In the case of Circinus and NGC 1365 the actual inclinations are given based on ionisation cone/molecular outflow estimates (Curran et al. 1999 and references within; Hjelm \& Lindblad 1996, respectively). The value for NGC 1365 is somewhat lower than expected for a Sy1.8, although Hjelm \& Lindblad (1996) class this as Sy1.5

\begin{tabular}{lrccc}
\hline Galaxy & $r$ & $i_{\text {mol }}$ & $i_{\text {main }}$ & Sy \\
\hline Circinus & 1 & $78^{\circ}$ & $65^{\circ}$ & $78^{\circ}$ \\
NGC 0034 & $<3$ & $\gtrsim 60^{\circ}$ & $70^{\circ}$ & Sy2 \\
NGC 1068 & $<2$ & $40^{\circ}-60^{\circ}$ & $35^{\circ}$ & Sy2 \\
NGC 1365 & $\lesssim 5$ & $30^{\circ}-40^{\circ}$ & $40^{\circ}$ & $35^{\circ} /$ Sy1.8 \\
NGC 1667 & $\lesssim 4$ & $\sim 30^{\circ}$ & $40^{\circ}$ & Sy2 \\
UGC 03374 & $\lesssim 3$ & $\lesssim 20^{\circ}$ & $40^{\circ}$ & Sy1.5 \\
NGC 2273 & $\sim 1$ & $20^{\circ}-50^{\circ}$ & $40^{\circ}$ & Sy2 \\
NGC 4593 & $\lesssim 1$ & $\lesssim 20^{\circ}$ & $40^{\circ}$ & Sy1 \\
Mrk 231 & $<7$ & $\lesssim 30^{\circ}$ & $40^{\circ}$ & Sy1 \\
NGC 5033 & - & $\gtrsim 60^{\circ}$ & $60^{\circ}$ & Sy1.9 \\
Mrk 273 & $\lesssim 4$ & $\approx 60^{\circ}$ & $75^{\circ}$ & Sy2 \\
NGC 5135 & $<5$ & $\approx 10^{\circ}$ & $46^{\circ}$ & Sy2 \\
NGC 5347 & - & - & $40^{\circ}$ & Sy2 \\
NGC 5548 & - & $\approx 70^{\circ}$ & $20^{\circ}$ & Sy1.5 \\
Arp 220 & $\lesssim 3$ & $40^{\circ}-50^{\circ}$ & $40^{\circ}$ & Sy2 \\
NGC 6814 & $\sim 1$ & $\lesssim 20^{\circ}$ & $20^{\circ}$ & Sy1.5 \\
NGC 7130 & $\lesssim 4$ & - & $20^{\circ}$ & Sy2 \\
NGC 7172 & $\gtrsim 1$ & $\sim 60^{\circ}$ & $60^{\circ}$ & Sy2 \\
NGC 7469 & $\sim 3$ & $\sim 10^{\circ}$ & $40^{\circ}$ & Sy1.2 \\
\hline
\end{tabular}

to be around a value of just under $20^{\circ}$, and since many of the inequalities occur at this value, we choose this for the median. For $i_{\text {mol }}-$ Sy, we use the same median and make the following approximations:

1. For Sy2s, a torus inclination $\gtrsim 70^{\circ}$,

2. for Sy1s, a torus inclination $\lesssim 20^{\circ}$, and

3. for Sy1.5s ${ }^{29}$, a torus inclination $\approx 45^{\circ}$.

The results are summarised in Fig. 11.

From Fig. 11 (left), we see that Sy2s tend to have their molecular ring/disc aligned with the main galaxy disc, whereas Sy1s tend to show a misalignment. However, the only Sy1 with an offset $\lesssim 20^{\circ}$ is actually a Sy1.5 (NGC 6814) and this value could quite possibly be

${ }^{29}$ The remaining sub-classes are estimated accordingly. 


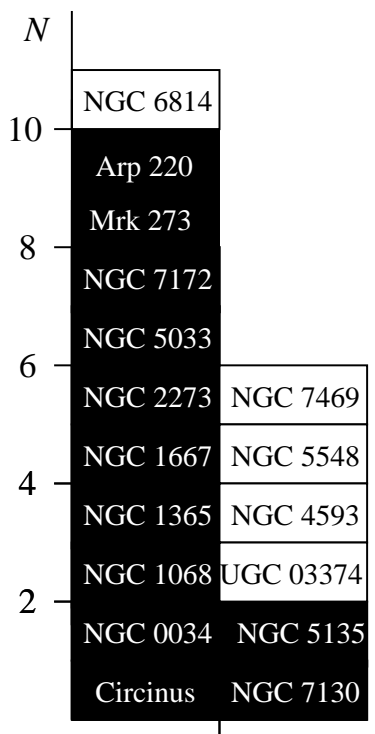

$<20>20$

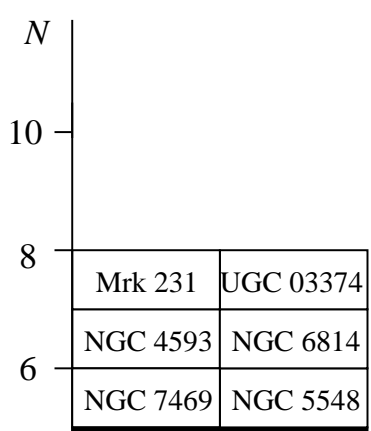

Mrk 273 NGC 5135

4 NGC 7172 NGC 2273

2 NGC 5033 NGC 7130 NGC 1365 NGC 1667 Circinus NGC 1068 $<20>20$

Fig. 11. Left: The offset in inclination (degrees) between the main galaxy disc and the molecular disc/ring. Right: The offset in inclination (degrees) between molecular ring and the obscuring torus. Black boxes represent Sy2s and white boxes Syls

$\gtrsim 20^{\circ}$, Table 2. Note that NGC 7130 (Sy2) is a borderline case with an offset of less than $20^{\circ}$ being quite feasible. Also, possibly consistent with our results, in NGC 1068 Schinnerer et al. (1999) suggest that the molecular disc has a higher inclination than the main disc, Table 2. Concerning the alignment between the dusty torus and the molecular ring, there appears to be no dependence on Seyfert type from our very approximate statistics, although for the sample (except NGCs 1667 and $5135^{30}$ these seem to be aligned within $\approx 30^{\circ}$, Fig. 11 (right).

Our results partly confirm the findings of McLeod \& Rieke (1995); Maiolino \& Rieke (1995), i.e. the galactic disc is aligned with the molecular ring. As mentioned previously, we find this only to be strictly true for Sy2s, although an offset of $\gg 20^{\circ}$ is only seen for one of the whole sample (NGC 5548, in which the model was noted to be uncertain, Sect. 3.13 $)^{31}$. Consistent with their results, from Table 2 we find that there is a (slight) tendency for Sy1s to be located in galaxies of low inclination and for Sy2s in galaxies of higher inclination, although intermediate inclinations seem to be favoured by both types.

\footnotetext{
30 And possibly UGC 03374 , NGCs 2273, 5347, 6814 and 7130. Note that these may still be approximately aligned with the molecular ring as geometrically thick tori could obscure at considerably lower inclinations than $70^{\circ}$ (Krolik \& Begelman 1988; Wilson \& Tsvetanov 1994; Maiolino \& Rieke 1995; Capetti et al. 1996)

31 Also possibly in NGC 5347, where the model is also uncertain (Sect. 3.12).
}

Regarding the relative aspects of the ring and torus, perhaps contrary to McLeod \& Rieke (1995); Maiolino \& Rieke (1995) we find that most tori tend to be aligned with the galactic disc (Table 2), although our result does appear to be consistent with that of Keel (1980); Lawrence \& Elvis (1982), and more recently, Wilson \& Tsvetanov (1994); Capetti et al. (1996). This suggests, since there are no large $\left(\gg 20^{\circ}\right)$ misalignments, that the conservation of angular momentum holds each structure approximately coplanar with its neighbour, i.e. $i_{\text {main }}-i_{\text {mol }} \lesssim 30^{\circ}$ and $i_{\text {mol }}-$ Sy $\lesssim 30^{\circ}$. Concerning particular examples, Gallimore et al. (1999) find from a sample of 13 Seyfert galaxies that the neutral atomic gas is distributed in a $100-$ pc scale rotating disc which has its axis aligned with that of the host galaxy and Greenhill et al. (1997) find that the masing disc in the Sy2 NGC 4945 has the same position angle as the host disc.

\section{Summary}

We have used the observational results of Curran et al. (2000) in order to investigate the possibility that there may generally be more molecular gas in type 2 than in type 1 Seyfert galaxies. Whereas our results may be consistent with those of Maiolino et al. (1997) in that we may find no difference in the mean CO/FIR luminosity ratio between the two classes, for sources in which $L_{\mathrm{FIR}} \sim 10^{11} L_{\odot}$, for less luminous Seyferts we find that the ratio in type 2 may be at least three times that in type 1 sources. This factor disappears when only the CO within the beam for the near-by $\left(L_{\mathrm{FIR}} \sim 10^{10} L_{\odot}\right)$ sources is considered. This possibly implies that the additional $\mathrm{CO}$ in the near-by type 2 sample may not be associated with the bulk of the FIR (or $\mathrm{HCN}^{32}$, Curran et al. 2000) emission. Could this additional CO trace the reservoir of molecular gas required to power the star-burst activity, which is lacking in type 1 Seyferts (Heckman 1987; Pogge 1989; Moorwood 1996; Hunt et al. 1997; Oliva et al. 1999)? Referring to Fig. 8 of Curran et al. (2000), this could well be the case; the CO/FIR luminosity ratio may generally decrease with increasing FIR luminosity ${ }^{33}$, and since in type 1 Seyferts a higher fraction of the luminosity must arise from the un-obscured AGN (e.g. Lawrence \& Elvis 1982), we suggest that a higher fraction of the FIR flux in type 2 (cf. type 1) Seyferts arises from star-burst activity. Although this contribution may decrease with increasing $L_{\text {FIR }}$ it will always constitute a greater portion of the FIR flux than in the type 1 Seyferts $^{34}$.

\footnotetext{
32 From Table 1 we find that $L_{\mathrm{HCN}} / L_{\mathrm{FIR}}(\mathrm{Sy} 2) \approx$ $L_{\mathrm{HCN}} / L_{\mathrm{FIR}}(\mathrm{Sy} 1) \approx 10^{-9} \mathrm{~K} \mathrm{~km} \mathrm{~s}^{-1} \mathrm{kpc}^{2} L_{\odot}^{-1}$.

33 We would expect this ratio to be independent of the FIR luminosity were the FIR emission arising mainly from young stars.

${ }^{34}$ Like the FIR, a significant proportion of the HCN emission could come from the AGN (Curran et al. 2000 and references
} 
From the CO detections we have also modelled the molecular gas distribution according to the molecular ring in the Circinus galaxy. From our results we conclude that:

1. For the sample the molecular ring does appear to be share a similar inclination as the galactic disc, although this alignment is tighter for type 2 Seyferts;

2. Type 2 nuclei show a slight preference for edge-on galaxies and type 1s for face-on systems, and in most cases, as with the ring and the main disc, the torus and ring tend to be aligned within $\approx 30^{\circ}$. This result could, however, be misleading if the $100 \mathrm{pc}$-scale ring contributes to the obscuration (Maiolino \& Rieke 1995).

Could the alignment between the host and ring in type 2 Seyferts be related to a greater molecular gas abundance? Curran et al. (1999) have shown that the orientation of the molecular ring should have little effect on the observed luminosity of the $\mathrm{CO}$ for our sample, but if there is indeed a gas deficit in type 1 Seyferts, perhaps it is due to a reduced gas flow causing instabilities and allowing the molecular ring to be further departed from the galactic plane. Since there appear to be larger gas concentrations in barred galaxies (Sakamoto et al. 1998) and bars may be more common in type 2 Seyferts (Pogge 1989), we suggest that the reservoir of gas which appears to be present in these galaxies may provide the stability necessary to hold the molecular ring coplanar to the galactic disc. Further evidence for alignment is given by the results of Maiolino et al. (1997); Papadopoulos \& Seaquist (1998) who attribute the excess of star-burst activity in type 2 Seyferts to the material in the obscuring torus still being funnelled down from the global scale via the 100 pc-scale ring ${ }^{35}$, thus suggesting that the torus may be but a dense component of the molecular ring (Shlosman et al. 1990; Friedli \& Martinet 1993; Shaw et al. 1993; Wilson \& Tsvetanov 1994; Fosbury et al. 2000; Conway 1999; Dopita et al. 1998; van Langevelde et al. 2000). This scenario would suggest that, in general, type 1 nuclei are more evolved than their "edge-on" counterparts; a notion supported by Blandford (1990); Maiolino et al. (1997); Rigopoulou et al. (1997); Dopita (1998); Papadopoulos \& Seaquist $(1998)^{36}$.

The ideas presented here are of course highly speculative, although it is hoped that the results from the model prove useful in guiding future interferometric observations.

within). Kohno et al. (1999) attribute the HCN luminosity in Seyferts to the obscuration and so a deficit in star-burst activity in type 1 nuclei may not significantly affect the observed HCN luminosity. This possibly explains why the CO luminosities differ while the HCN luminosities are similar between the two classes.

${ }^{35}$ Tacconi et al. (1999b) have noted several couplings of the $100-$ pc scale disc to the larger scale gas in the luminous merger NGC 6240.

36 The transformation of a type 2 to a type 1 Seyfert has recently been noted by Aretxaga et al. (1999).
Acknowledgements. I would like to thank S. Aalto whose idea was to study the relative gas abundances and also L.E.B. Johansson and M. Olberg for their help. I would also like to thank the referee R. Antonucci for his very helpful comments. This research has made use of the NASA/IPAC Extragalactic Database (NED) which is operated by the Jet Propulsion Laboratory, California Institute of Technology, under contract with the National Aeronautics and Space Administration.

\section{References}

Antonucci R.R.J., Miller J.S., 1985, ApJ 297, 621

Antonucci R.R.J., 1993, ARA\&A 31, 473

Aretxaga I., Joguet B., Kunth D., Melnick J., Terlevich R.J., 1999, ApJ 519, L123

Bake A.J., Scoville N.Z., 1998, Amer. Astron. Soc. Meet. 192, 3605

Bergman P., Aalto S., Black J.H., Rydbeck G., 1992, A\&A 265, 403

Blandford R.D., Netzer H., Woltjer L., Courvoisier T., Mayor M., 1990, Active Galactic Nuclei. Springer-Verlag, Berlin

Bryant P.M., Scoville N.Z., 1996, ApJ 457, 678

Burbridge E.M., Burbridge G.R., 1960, ApJ 132, 30

Capetti A., Axon D.J., Macchetto F., Sparks W.B., Boksenberg A., 1996, ApJ 469, 554

Conway J., 1999, HI Absorption from a Circumnuclear TORUS in the Hidden Quaser Cygnus A, in: Carilli C., Radford S., Menton K., Langston G. (eds.), Highly Redshifted Radio Lines, ASP Conf. Ser., p. 259

Curran S.J., Aalto S., Booth R.S., 2000, A\&AS 141, 193

Curran S.J., Johansson L.E.B., Rydbeck G., Booth R.S., 1998, A\&A 338, 863

Curran S.J., Rydbeck G., Johansson L.E.B., Booth R.S., 1999, A\&A 344, 767

Curran S.J., Koribalski B., Haynes R.F., Krause M., Johansson L.E.B., 2000a, A\&A (in preparation)

Curran S.J., Rydbeck G., Johansson L.E.B., 2000b, A\&A (in preparation)

Curran S.J., 1998, Licentiate thesis, Chalmers University of Technology

Curran S.J., 2000, Ph.D. Thesis, Chalmers University of Technology

Dahlem M., Golla G., Whiteoak J.B., et al., 1993, A\&A 270, 29

Dopita M.A., Heisler C., Lumsden S., Bailey J., 1998, ApJ 498, 570

Dopita M.A., 1998, Proc. Astron. Soc. Aust. 14, 230

Downes D., Solomon P.M., 1998, ApJ 507, 615

Edelson R.A., 1987, ApJ 313, 651

Elmouttie M., Krause M., Haynes R.F., Jones K.L., 1998a, MNRAS 300, 1119

Elmouttie M., Koribalski B., Gordon S., et al., 1998b, MNRAS 297, 49

Fernandes Roberto Cid J., Terlevich R., 1995, MNRAS 272, 423

Fosbury R.A.E., Vernet J., Villar-Martin M., et al., 2000, Optical Continuum Structure of Cygnus A, in: Best P., Lehnert M. (eds.), KNAW colloqium on: The Most Distant Radio Galaxies. Reidel, Amsterdam (in press)

Freeman K.C., Karlsson B., Lyngå G., et al., 1977, A\&A 55, 445 
Friedli D., Martinet L., 1993, A\&A 277, 27

Gallimore J.F., Baum S.A., O'Dea C.P., Pedlar A., Brinks E., 1999, ApJ 524, 684

Greenhill L.J., Moran J.M., Herrnstein J.R., 1997, ApJ 481, L23

Güsten R., 1989, Gas and Dust in the Inner Few Degrees of the Galaxy, in: Morris M. (ed.) IAU Symposium No. 136: The Center of the Galaxy. Reidel, Dordrecht, p. 89

Heckman T.M., Beckwith S., Blitz L., Skrutskie M., Wilson A.S., 1986, ApJ 305, 157

Heckman T.M., Blitz L., Wilson A.S., Armus L., Miley G.K., 1989, ApJ 342, 735

Heckman T.M., 1987, Star Formation in Active Galaxies and Quasars, in: Thuan T.X., Montmerle T,, Van J.T.T. (eds.), Starbursts and Galaxy Evolution. Éditions Frontières, Paris, p. 381

Hjelm M., Lindblad P., 1996, A\&A 305, 727

Hunt L.K., Malkan M.A., Salvati M., et al., 1997, ApJS 108, 229

Irwin J.A., Sofue Y., 1992, ApJ 396, L75

Keel W.C., 1980, AJ 85, 198

Kenney J.D.P., Wilson C.D., Scoville N.Z., Devereux N.A., Young J.S., 1992, ApJ 395, L79

Kenney J., 1996, Molecular Gas in the Central Regions of Barred Galaxies, in: Buta R., Crocker D.A., Elmegreen B.G. (eds.), IAU Colloquium No. 157: Barred Galaxies. ASP Conf. Ser., San Francisco, p. 150

Kohno K., Kawabe R., Tosaki T., Okumura S.K., 1996, ApJ 461, L29

Kohno K., Kawabe R., Vila-Vilaró B., 1999, NMA Survey of $\mathrm{CO}$ and HCN Emission from Nearby Active Galaxies, in: Ossenkopf V., Stutzki J., Winnewisser G. (eds.), Proceedings of the 3rd Cologne-Zermatt Symposium, "The Physics and Chemistry of the Interstellar Medium", p. 2251 (in press)

Krolik J.H., Begelman M.C., 1988, ApJ 329, 702

Lawrence A., Elvis M., 1982, ApJ 256, 410

Lindblad P.A.B., Lindblad P.O., Athanassoula E., 1996, ApJ 313, 65

Lindblad P.O., 1978, On the Nuclei of the Barred Galaxies NGC 1365 and NGC 1512, in: Reiz A., Andersen T. (eds.), Astronomical Papers Dedicated to Bengt Strömgren. Copenhagen University Observatory, Copenhagen, p. 404

Lonsdale C.J., Helou G., Good J.C., Rice W., 1985, Cataloged Galaxies and Quasars Observed in the IRAS Survey. Jet Propulsion Laboratory, Pasadena

Maiolino R., Rieke G.H., 1995, ApJ 454, 95

Maiolino R., Ruiz M., Rieke G.H., Papadopoulos P., 1997, ApJ 485,552

Maloney P., Black J.H., 1988, ApJ 325, 389

Maloney P., 1990, Mass Determinations from CO Observations, in: H.A. Thronson J., Shull J.M. (eds.), The Interstellar Medium in Galaxies. Kluwer, Dordrecht, p. 493

Marconi A., Moorwood A.F.M., Origlia L., Oliva E., 1994, ESO Messenger 78, 20

Mauersberger R., Henkel C., Whiteoak J.B., Chin Y.N., Tieftrunk A.R., 1996, A\&A 309, 705

McLeod K.K., Rieke G.H., 1995, ApJ 441, 96
Meurs E.J.A., Wilson A.S., 1984, A\&A 136, 227

Moorwood A.F.M., 1996, Space Sci. Rev. 77, 303

Myers S.T., Scoville N.Z., 1987, ApJ 312, L39

Neufeld D.A., Maloney P.R., 1995, ApJ 447, L17

Oliva E., Origlia L., Maiolino R., Moorwood A.F.M., 1999, A\&A 350, 9

Osterbrock D.E., Shaw R.A., 1988, ApJ 327, 89

Papadopoulos P.P., Seaquist E.R., 1998, ApJ 492, 521

Papadopoulos P.P., Seaquist E.R., Scoville N.Z., 1996, ApJ 465,173

Peterson B.M., 1997, An Introduction to Active Galactic Nuclei. Cambridge University Press, Cambridge

Planesas P., Gomez-Gonzalez J., Martin-Pintado J., 1989, A\&A 216, 1

Plante R.L., Lo K.Y., Roy J.-R., Martin P., Noreau L., 1991, ApJ 381, 110

Pogge R.W., 1989, ApJ 345, 730

Rigopoulou D., Papadakis I., Lawrence A., Ward M., 1997, A\&A 327, 493

Sahai R., Sundin M., Claussen M.J., Rickard L.J., 1991, A survey of CO emission from Southern Seyfert Galaxies, in: Combes F., Casoli F. (eds.), IAU Symposium No. 146: Dynamics of Galaxies and Their Molecular Cloud Distributions. Reidel, Dordrecht, p. 327

Sakamoto K., Okumura S.K., Ishizuki S., Scoville N.Z., 1998, BAAS 192, 6609

Sandqvist A., Jörsäter S., Lindblad P., 1995, A\&A 295, 585

Sandqvist A., 1999, A\&A 343, 367

Schinnerer E., Eckart A., Tacconi L.J., 1999, ApJ 523, L5

Scoville N.Z., Yun M.S., Bryant P.M., 1997, ApJ 484, 702

Shaw M.A., Combes F., Axon D.J., Wright G.S., 1993, A\&A 273,31

Shier L.M., Rieke M.J., Rieke G.H., 1994, ApJ 433, L9

Shlosman I., Begelman M.C., Frank J., 1990, Nat 345, 679

Sofue Y., 1991, PASJ 43, 671

Strong A.W., et al., 1988, A\&A 207, 1

Tacconi L.J., Genzel R., Blietz M., et al., 1994, ApJ 426, L77

Tacconi L.J., Gallimore J.F., Schinnerer E., Genzel R., Downes D., 1996, BAAS 189, 906

Tacconi L., Genzel R., Schinnerer E., Eckart A., Tecza M., 1999a, Molecular Gas Disks in Nearby AGN and Mergers: a glimpse into high- $z$ studies with ALMA, in: Science with the Atacama Large Millimeter Array (ALMA), Associated Universities, Inc., held October 6-8, 1999 at Carnegie Institution of Washington, p. E6

Tacconi L.J., Genzel R., Tecza M., et al., 1999b, ApJ 524, 732

Tadhunter C., Tsvetanov Z., 1989, Nat 341, 422

Teuben P.J., Sanders R.H., Atherton P.D., van Albada G.D., 1986, MNRAS 221, 1

van Langevelde H.J., Pihlström Y.M., Conway J.E., Jaffe W., Schilizzi R.T., 2000, A\&A 345, L45

Veilleux S., Bland-Hawthorn J., 1997, ApJ 479, L105

Wilson A.S., Tsvetanov Z.I., 1994, AJ 107, 1227

Wilson A.S., Helfer T.T., Haniff C.A., Ward M.J., 1991, ApJ 381, 79

Wilson A.S., Ward M.J., Haniff C.A., 1988, ApJ 334, 121

Young J.S., et al., 1995, ApJS 98, 219

Yun M.S., Scoville N.Z., 1995, ApJ 451, L45 